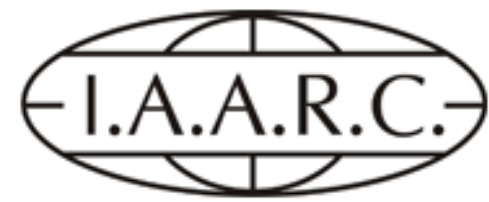

Home

About IAARC

Membership

News and Events

International Association for
Automation and Robotics in Construction

I AARC PUPLICATI ONS IAARC Awards Contact Sitemap

\title{
Fast terminal sliding mode control for gantry cranes
}

Ansu Man Singh, Van Truong Hoang and Q. P. Ha

Pages 437-443 (2016 Proceedings of the 33rd ISARC, Auburn, USA)

Cranes remain a vital tool for the construction of infrastructure such as buildings, bridges, etc. Recently, there has been renewed interest in crane automation in dealing with concerns on safety and possible performance degradation due to system uncertainties and disturbances. One potential solution to the problem is the use of robust techniques based on the Sliding Mode Control (SMC) methodology. Much research has been conducted to design controllers based on linear sliding surfaces, aiming at achieving the desired control performance in finite time. In this context, this paper proposes a control method, based on the Fast Terminal Sliding Mode (FTSM), to guarantee finite-time stability of the crane. To do that, we have derived a mathematical model of the crane using Lagrangian formulation with uncertainties as bounding functions. Then, sliding surfaces based on the hierarchical sliding mode are defined, and a control law is derived using the Lyapunov stability theory. The hierarchical sliding surfaces consist of two layers. The first layer include sliding functions based on FTSM to enable faster convergence of the system to equilibrium. This can have advantages in high precision tracking applications. In the second-layer, the sliding surface is designed from the linear combination of the first layer sliding functions. Also, we have given a proof of the stability of the system in finite time. Extensive simulation results show the proposed controller based on FTSM can achieve higher performance in stabilizing the swinging load of a gantry crane. Laboratorial experiments have been conducted to verify the obtained results in terms of the superior convergence time and improved performance over conventional SMC.

Keywords: Machine Control Systems, Mechatronics, hoist control, Automation and Robotics

Download fulltext 


\title{
Fast terminal sliding mode control for gantry cranes
}

\author{
Ansu Man Singh, Van Truong Hoang and Q. P. Ha \\ School of Electrical, Mechanical and Mechatronic Systems, \\ University of Technology Sydney, Australia \\ E-mail: AnsuMan.Singh@uts.edu.au, VanTruong.Hoang@student.uts.edu.au, Quang.Ha@uts.edu.au
}

\begin{abstract}
Cranes remain a vital tool for the construction of infrastructure such as buildings, bridges, etc. Recently, there has been renewed interest in crane automation in dealing with concerns on safety and possible performance degradation due to system uncertainties and disturbances. One potential solution to the problem is the use of robust techniques based on the Sliding Mode Control (SMC) methodology. Much research has been conducted to design controllers based on linear sliding surfaces, aiming at achieving the desired control performance in finite time. In this context, this paper proposes a control method, based on the Fast Terminal Sliding Mode (FTSM), to guarantee finite-time stability of the crane. To do that, we have derived a mathematical model of the crane using Lagrangian formulation with uncertainties as bounding functions. Then, sliding surfaces based on the hierarchical sliding mode are defined, and a control law is derived using the Lyapunov stability theory. The hierarchical sliding surfaces consist of two layers. The first layer include sliding functions based on FTSM to enable faster convergence of the system to equilibrium. This can have advantages in high precision tracking applications. In the second-layer, the sliding surface is designed from the linear combination of the first layer sliding functions. Also, we have given a proof of the stability of the system in finite time. Extensive simulation results show the proposed controller based on FTSM can achieve higher performance in stabilizing the swinging load of a gantry crane. Laboratorial experiments have been conducted to verify the obtained results in terms of the superior convergence time and improved performance over conventional SMC.
\end{abstract}

\footnotetext{
Keywords -

Machine Control Systems, Mechatronics, hoist control, Automation and Robotics
}

\section{Introduction}

Cranes have a pivotal role in the construction of high-rise buildings, bridges, etc. Automation and control of cranes has been the subject of many studies to address safety and performance in the presence of parametric uncertainties and external disturbances.

Problems of uncertainties and external disturbances in the cranes can be solved by employing robust controllers, for which the Sliding Mode Control (SMC) method is very promising. Many published studies have shown the synthesis of such controllers. For instance, optimal sliding mode is proposed for trajectory tracking of offshore cranes [1] or second-order sliding mode is applied for vibration suppression in smart structures [2] to address the problem of uncertainties and external disturbances in these systems. Similarly, sliding surfaces incorporating the load swing dynamics along with an adaptive method to tune the controller's gain are presented in [3] for container cranes. Studies on the subject have mostly focused on the controllers that utilize linear sliding surfaces. By using these control approaches, convergence to the system equilibrium is asymptotic, and hence, the settling time of the crane operations may be not fast enough. Therefore, it is worth looking for a SMC scheme with a finite time reaching phase.

To enable finite time reachability of the system equilibrium while also guaranteeing robustness in the system, terminal sliding mode (TSM) has been proposed in [4] and applied to many mechanical systems [5]. However, there are issues with performance, particularly in the high-precision applications, due to the slower response of TSM when the system's states are far from equilibrium under a constrained control force. This paper presents a faster, finite-time stable, and robust controller for gantry cranes. The controller is based on Fast Terminal Sliding Mode (FTSM), a variant of TSM. The idea of FTSM is to provide faster convergence both at a distance from and at a close range 
of the equilibrium [6]. Many published studies havewhere coordinates $\boldsymbol{q}=\left[\begin{array}{ll}q_{x} & q_{\theta}\end{array}\right]^{\top}=\left[\begin{array}{ll}x & \theta\end{array}\right]^{\top} \in \mathbb{R}^{2}$, reported the application of FTSM on the fully-actuated systems in robotics and automation applications. However, to date, no studies have shown its design and implementation on underactuated systems such as cranes. The methodology used in the synthesis of the proposed controller is as follows: first, the two layer sliding functions are defined based on hierarchical SMC. The first layer consists of FTSM manifolds. The second layer is the linear combination of the first layer sliding functions. Then, the Lyapunov stability theory is used to derive the control law for the system.

The organization of this paper is as follows. A mathematical model for the gantry cranes is obtained in the next section, followed by Section 3 for the derivation of the proposed controller and its stability analysis. Simulation and experimental results are presented in Sections 4 and 5, respectively. Finally, the paper conclusion arrives with some recommendations.

\section{Mathematical model of gantry cranes}

The structure of a gantry type crane is depicted in Figure 1. It consists of a cart of mass $m_{c}$, a hoisting mechanism through a rope of length $l$, and a payload of mass $m_{p}$. The position of the cart and the swing angle of the payload are represented by $x$ and $\theta$, respectively. In this paper we have made following assumptions:

- the payload consists of a point mass

- the stiffness of the hoisting rope is negligible.

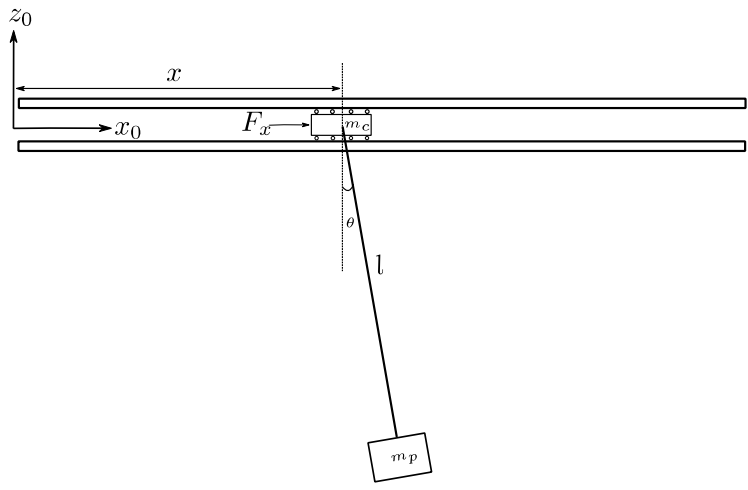

Figure 1. Structure of a gantry crane.

Using the Lagrangian formulation [7], the dynamic model of the gantry crane can be represented as,

$$
M(\boldsymbol{q}) \ddot{\boldsymbol{q}}+C(\boldsymbol{q}, \dot{\boldsymbol{q}})+g(\boldsymbol{q})=B u,
$$

$$
\begin{aligned}
M(\boldsymbol{q})= & {\left[\begin{array}{ll}
m_{11} & m_{12} \\
m_{21} & m_{22}
\end{array}\right] } \\
& =\left[\begin{array}{cc}
m_{c}+m_{p} & m_{p} l \cos (\theta) \\
m_{p} l \cos (\theta) & m_{p} l^{2}
\end{array}\right]
\end{aligned}
$$

is the inertial matrix,

$C(\boldsymbol{q}, \dot{\boldsymbol{q}})=\left[\begin{array}{c}-m_{p} l \sin (\theta) \dot{\theta} \\ 0\end{array}\right]$

is the Coriolis matrix, and

$g(\boldsymbol{q})=\left[\begin{array}{c}0 \\ -m_{p} g l \sin (\theta)\end{array}\right]$

is the gravitational term. The signal $u$ is the control input with $B=\left[\begin{array}{ll}1 & 0\end{array}\right]^{\top}$ being the input matrix. Equation (1) can be formulated in the following form

$\ddot{q}_{x}=f_{x}(\boldsymbol{q}, \dot{\boldsymbol{q}})+b_{x}(\boldsymbol{q}) u+\sigma_{x}(\boldsymbol{q})$
$\ddot{q}_{\theta}=f_{\theta}(\boldsymbol{q}, \dot{\boldsymbol{q}})+b_{\theta}(\boldsymbol{q}) u+\sigma_{\theta}(\boldsymbol{q})$,

where,

$f_{x}(\boldsymbol{q}, \dot{\boldsymbol{q}})=\frac{m_{p}^{2} l^{2} \sin \theta(l \dot{\theta}-g \cos \theta)}{\left(m_{p}+m_{c}\right) m_{p} l^{2}-m_{p}^{2} l^{2} \cos ^{2} \theta}$,

$f_{\theta}(\boldsymbol{q}, \dot{\boldsymbol{q}})=\frac{m_{p} l \sin \theta\left\{\left(m_{p}+m_{c}\right) g-m_{p} l \cos \theta \dot{\theta}\right\}}{\left(m_{p}+m_{c}\right) m_{p} l^{2}-m_{p}^{2} l^{2} \cos ^{2} \theta}$,

$b_{x}(\boldsymbol{q})=\frac{m_{p} l^{2}}{\left(m_{p}+m_{c}\right) m_{p} l^{2}-m_{p}^{2} l^{2} \cos ^{2} \theta}$,

$b_{\theta}(\boldsymbol{q})=\frac{-m_{p} l \cos \theta}{\left(m_{p}+m_{c}\right) m_{p} l^{2}-m_{p}^{2} l^{2} \cos ^{2} \theta}$.

In (2), the lumped terms $\sigma_{x}(\boldsymbol{q})$ and $\sigma_{\theta}(\boldsymbol{q})$ represents the system uncertainties of the system as well as external disturbances. They are assumed to be bounded, i.e. $\left|\sigma_{x}(\boldsymbol{q})\right| \leq \mu_{x}$ and $\left|\sigma_{\theta}(\boldsymbol{q})\right| \leq \mu_{\theta}$.

\section{Control system design}

\subsection{Fast Terminal Sliding Mode}

Sliding function for FTSM is given by

$s=\dot{x}+\beta x^{q / p}+\alpha x$,

where $\alpha>0, \beta>0, q>0$, and $p>0$. Moreover, $q$ and $p$ are odd integers such that $q<p$. In the sliding surface $s=0$ the state $x$ in (3) reaches the equilibrium point $x=0$ in finite time, also known as the reaching 
time, is given by

$t_{s}=\frac{p}{\alpha(p-q)} \ln \left(1+\frac{\alpha x_{0}^{(p-q) / p}}{\beta}\right)$,

where $x_{0}=x(0)$ is the initial condition. The trajectory of the system in the sliding surface $s=0$ can be obtained as

$x(t)= \begin{cases}\operatorname{sgn}\left(x_{0}\right)\left\{\frac{\left(\alpha x_{0}^{(p-q) / p}+\beta\right) e^{-\frac{\alpha(p-q)}{p} t}-\beta}{\alpha}\right\}^{\frac{p}{p-q}} & t \leq t_{s} \\ 0 & t>t_{s}\end{cases}$

The difference between sliding surfaces of FTSM (3) and TSM is the additional linear attractor $\alpha x$ in addition to terminal attractor $\beta x^{q / p}$ [8]. The presence of the linear attractor is to make the state of the FTSM sliding surface converges to equilibrium at a faster rate compared to TSM [6] when the initial state is far from the equilibrium point.

\subsection{Sliding surface design for the crane}

The position error and the swing angle error of the crane are defined respectively as $e_{x}=x-x_{d}$ and $e_{\theta}=\theta$. Since the crane is an underactuated system, it is intuitive to design the first layer with two sliding functions:

$s_{1}=\dot{e}_{x}+\beta_{x} e_{x}^{q_{1} / p_{1}}+\alpha_{x} e_{x}$

$s_{2}=\dot{e}_{\theta}+\beta_{\theta} e_{\theta}^{q_{2} / p_{2}}+\alpha_{\theta} e_{\theta}$,

where $p_{1}, q_{1}, p_{2}$, and $q_{2}$ are odd integers such that $\frac{q_{1}}{p_{1}}<1$ and $\frac{q_{2}}{p_{2}}<1$. Also, for coefficients of the terminal and linear attractors, scalars $\beta>0$ and $\alpha>0$. Now, the second layer sliding function is defined as,

$S=k_{1} s_{1}+k_{2} s_{2}$

where $k_{1}>0$ and $k_{2}>0$, which is the linear combination of first layer sliding functions. This strategy is known as the hierarchical sliding mode [9].

\subsection{Stability analysis of the sliding surfaces}

In the following, we design a control input for the system that guarantees the stability of the system and the convergence of the sliding surface $S=0$. Let us first define a Lyapunov function for $S$ as,

$V=\frac{1}{2} S^{2}$

Taking time derivative, one can get
$\dot{V}=S \dot{S}$

The time derivative of the of Equation (7) is given by

$$
\begin{aligned}
\dot{S}= & k_{1}\left(\ddot{e}_{x}+\beta_{x} \frac{q_{1}}{p_{1}} e_{x}^{q_{1} / p_{1}-1} \dot{e}_{x}+\alpha_{x} \dot{e}_{x}\right) \\
& +k_{2}\left(\ddot{e}_{\theta}+\beta_{\theta} \frac{q_{2}}{p_{2}} e_{\theta}^{q_{2} / p_{2}-1} \dot{e}_{\theta}+\alpha_{\theta} \dot{e}_{\theta}\right)
\end{aligned}
$$

or,

$$
\begin{aligned}
\dot{S}= & k_{1}\left\{f_{x}(\boldsymbol{q}, \dot{\boldsymbol{q}})+b_{x}(\boldsymbol{q}) u\right. \\
& \left.+\beta_{x} \frac{q}{p} e_{x}^{q_{1} / p_{1}-1} \dot{e}_{x}+\alpha_{x} \dot{e}_{x}-\ddot{x}_{d}+\sigma_{x}(\boldsymbol{q})\right\} \\
& +k_{2}\left\{f_{\theta}(\boldsymbol{q}, \dot{\boldsymbol{q}})+b_{\theta}(\boldsymbol{q}) u\right. \\
& \left.+\beta_{\theta} \frac{q}{p} e_{\theta}^{q_{2} / p_{2}-1} \dot{e}_{\theta}+\alpha_{\theta} \dot{e}_{\theta}+\sigma_{\theta}(\boldsymbol{q})\right\}
\end{aligned}
$$

or,

$$
\begin{aligned}
\dot{S}= & k_{1}\left\{f_{x}(\boldsymbol{q}, \dot{\boldsymbol{q}})+\beta_{x} \frac{q}{p} e_{x}^{q_{1} / p_{1}-1} \dot{e}_{x}+\alpha_{x} \dot{e}_{x}-\ddot{x}_{d}+\sigma_{x}(\boldsymbol{q})\right\} \\
& +k_{2}\left\{f_{\theta}(\boldsymbol{q}, \dot{\boldsymbol{q}})+\beta_{\theta} \frac{q}{p} e_{\theta}^{q_{2} / p_{2}-1} \dot{e}_{\theta}+\alpha_{\theta} \dot{e}_{\theta}+\sigma_{\theta}(\boldsymbol{q})\right\} \\
& +\left\{k_{1} b_{x}(\boldsymbol{q})+k_{2} b_{\theta}(\boldsymbol{q})\right\} u
\end{aligned}
$$

Now, let us propose the control input

$u=-\frac{1}{k_{1} b_{x}(\boldsymbol{q})+k_{2} b_{\theta}(\boldsymbol{q})}\left\{u_{1}+u_{2}+u_{3}\right\}$,

where,

$$
\begin{aligned}
& u_{1}=k_{1}\left(f_{x}(\boldsymbol{q}, \dot{\boldsymbol{q}})+\beta_{1} e_{x}^{q_{1} / p_{1}-1} \dot{e}_{x}+\alpha_{1} \dot{e}_{x}-\ddot{x}_{d}\right) \\
& u_{2}=k_{2}\left(f_{\theta}(\boldsymbol{q}, \dot{\boldsymbol{q}})+\beta_{2} e_{\theta}^{q_{2} / p_{2}-1} \dot{e}_{\theta}+\alpha_{2} \dot{e}_{\theta}\right) \\
& u_{3}=\eta \operatorname{sgn}(S)+K S,
\end{aligned}
$$

in which $\eta>0$ and $K>0$. By applying the input (11) to Equation (10) and after some arrangement, we obtain

$$
\begin{aligned}
\dot{V}=S \dot{S} & =S\left\{k_{1} \sigma_{x}(\boldsymbol{q})+k_{2} \sigma_{\theta}(\boldsymbol{q})-\eta \operatorname{sgn}(S)-K S\right\} \\
& =-K S^{2}-\eta|S|+\left\{k_{1} \sigma_{x}(\boldsymbol{q})+k_{2} \sigma_{\theta}(\boldsymbol{q})\right\} S \\
& =-K S^{2}-\eta|S|+\left\{k_{1} \sigma_{x}(\boldsymbol{q})+k_{2} \sigma_{\theta}(\boldsymbol{q})\right\}|S|
\end{aligned}
$$

where $D_{m}=k_{1} \sigma_{x}(\boldsymbol{q})+k_{2} \sigma_{\theta}(\boldsymbol{q})$.

If $\eta$ is chosen such that

$\eta>D_{m}=\sup \left(k_{1} \mu_{x}+k_{2} \mu_{\theta}\right)$,

then

$\dot{V}=-K S^{2}-\left(\eta-D_{m}\right)|S| \leq 0$. 
By applying the Lyapunov stability theory, it can be verified that the second layer sliding surface $S$ is convergent to zero. In [10],Wang et al. have proved that if the second layer sliding surface is driven to the origin; then the first layer sliding functions, i.e., $s_{1}$ and $s_{2}$, are also convergent to the origin, i.e. $s_{1}=0$ and $s_{2}=0$. Thus, following the same argument we can conclude that $e_{x}$ and $e_{\theta}$ reach the equilibrium in finite time as determined by (4).

\section{Simulation Results}

To validate the proposed method we used the gantry crane available in our control laboratory, as shown in Figure 2. The parameters of the crane and the design parameters of the proposed controller are provided in Table 1 . To select values for $p_{1}, q_{1}, p_{2}, q_{2}, \alpha_{x}, \alpha_{\theta}, \beta_{x}$, and $\beta_{\theta}$ we followed the conditions given in Section 3.3 and considered the reaching time expression for FTSM (4) for the required reaching time of the cart position and the swing angle. However, to optimize the values we did a number of trials in the simulation. Similarly, the values of $k_{1}$ and $k_{2}$ were chosen with some trialand-error steps during the simulation.

Table 1

\begin{tabular}{c|c}
\hline System parameters & Value \\
\hline$m_{p}$ & $2.24 \mathrm{~kg}$ \\
$m_{c}$ & $2.27 \mathrm{~kg}$ \\
$l$ & $0.5 \mathrm{~m}$ \\
$g$ & $9.8 \mathrm{~m} / \mathrm{s}^{2}$ \\
$\left|\sigma_{x}(q)\right|$ & $\leq 0.5 \mathrm{~m} / \mathrm{s}^{2}$ \\
$\left|\sigma_{\theta}(q)\right|$ & $\leq 0.5 \mathrm{rad} / \mathrm{s}^{2}$ \\
\hline & $\alpha_{x}=\alpha_{\theta}=0.1, \beta_{x}=\beta_{\theta}=0.4$ \\
Control parameters & $\eta=0.1, p_{1}=11$, \\
& $q_{1}=9, p_{2}=9, q_{2}=7$ \\
& $k_{1}=2, k_{1}=1, K=1$
\end{tabular}

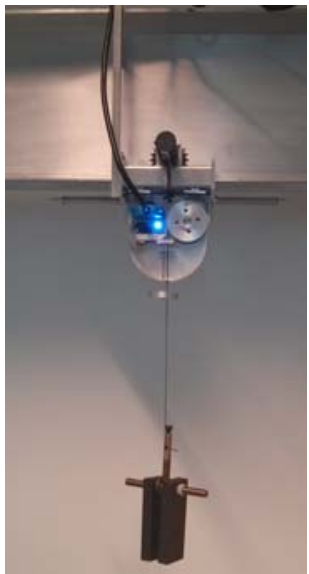

Figure 2: Gantry crane photo.
Figure 3 and Figure 4 show the simulation results of the cart position and the payload swing angle, respectively. The reference position for the cart is $1.5 \mathrm{~m}$. Both results show achievement of the equilibrium point within 6 sec. To judge the control performance, Figure 5 represents the sliding surfaces. It is clear from the plot that the second layer sliding function $S$ can reach $S=0$ within $3 \mathrm{sec}$ and the first layer sliding functions $s_{1}$ and $s_{2}$ approach the equilibrium asymptotically.

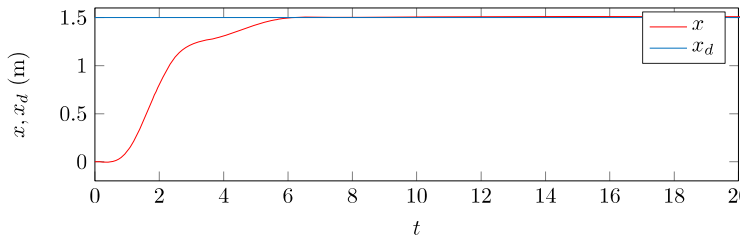

Figure 3: Cart position of the crane

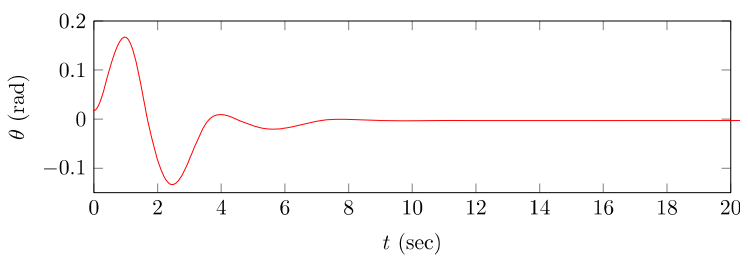

Figure 4: Swing angle of the payload

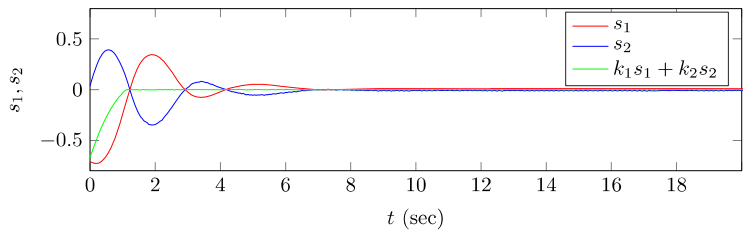

Figure 5: First and second layer sliding functions

Figure 6 shows the control input generated by the control system. The peak control force is around $12 \mathrm{~N}$. Chattering can be observed after the system reaches its final position. This undesired effect can be reduced by using the well-known saturated function.

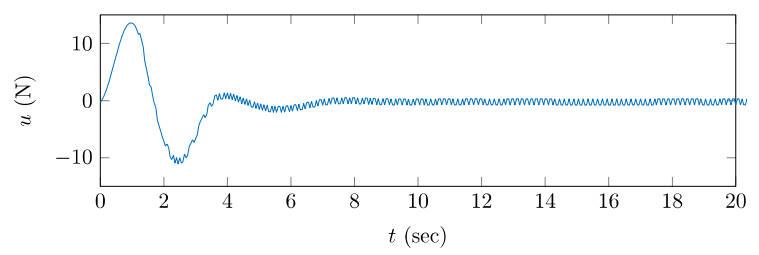

Figure 6: Control input to the plant

Figure 7 compares the result obtained from our proposed method and TSM. The cart reference for both the controller remains $1.5 \mathrm{~m}$ and the starting location is the origin. It is observed that the FTSM controller drives the cart to the set point in $6 \mathrm{sec}$ whereas it takes $7 \mathrm{sec}$ 
for the TSM. Therefore, a faster convergence is the clear advantage of the proposed method over TSM. Indeed, a faster settling of the control system would imply a higher efficiency of the construction or manufacturing automation processes that require pickand-place operations of a crane.

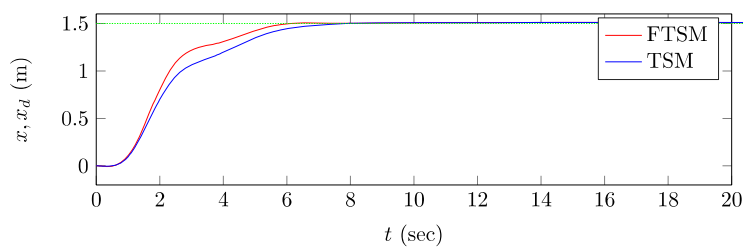

Figure 7: Comparison study of FTSM and TSM.

\section{Experimental setup and results}

To experimentally verify the proposed method, we implemented a control system for the crane of Figure 2 by using a STM32f411 Nucleo microprocessor board, as depicted in the Figure 8. The board consists of an ARM Cortex M4 processor with $512 \mathrm{~KB}$ of flash memory and $128 \mathrm{~KB}$ of RAM. It receives the feedback signals, i.e. position of cart and payload's swing angle, from the crane through a signal conditioning circuit. The purpose of the circuit is to provide interface between the crane and the microprocessor board. Similarly, the control signal generated by the board is fed to crane through a Digital to Analog Converter (DAC) AD5726 IC. The DAC IC is connected with the board using SPI interface. The board is also connected to a PC via an UART interface to collect data from the crane's sensors for post processing.

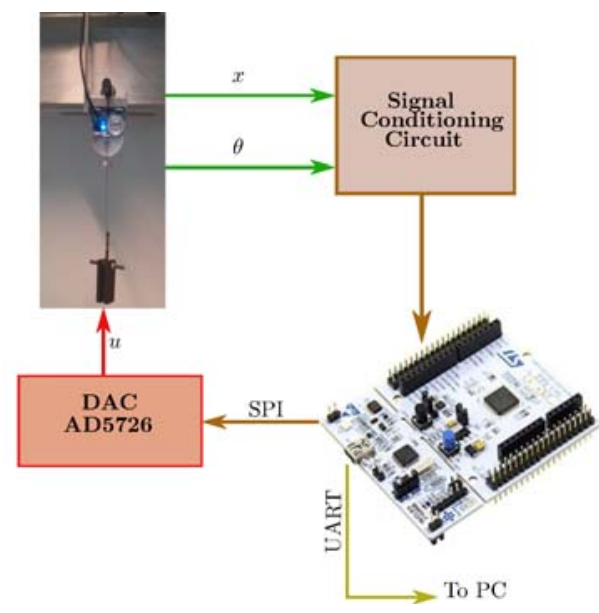

Figure 8: Experimental setup

The Nucleo board runs the FreeRTOS operating system (OS) and the proposed controller is implemented as an OS's stack. The feedback signals from the crane are sampled at the rate of $10 \mathrm{kHz}$. Similarly the controller generates the control signal every $0.01 \mathrm{sec}$.

Figure 9 and Figure 10 show responses of the cart position and payload swing angle, respectively. The sampling frequency for the graphs is $10 \mathrm{~Hz}$. The reference position for the cart, during the experiments, was set to $1.5 \mathrm{~m}$. The initial position of the cart is $0.1 \mathrm{~m}$, and reaches the desired location with the steady state error less than $0.05 \mathrm{~m}$.

Initially the swing angle of the payload reaches up to 11 degrees. The oscillation, however, decreases to less than 3 degrees. The obtained results are quite coincident with those from the simulation. There are many reasons for the oscillation. One of the reasons is the uncompensated dynamics of the motor in the model of the crane, i.e. equation (1). Another is with the approximation. Indeed, the controller requires position and velocity feedback from the system, i.e. $\boldsymbol{q}$ and $\dot{\boldsymbol{q}}$. However, only position feedback is available. Therefore, for the experiment, we developed an estimator of the velocity using an approximate differentiator as

$\dot{\boldsymbol{q}}=\frac{\boldsymbol{q}(t)-\boldsymbol{q}(t-T)}{T}$,

where $T$ is the sampling period of the controller. It should be noted that (13) does not provide an accurate estimation of the velocity, particularly of the swing angle. Therefore, by using another sensor or a more accurate estimation method, performance of our proposed control method can be enhanced.

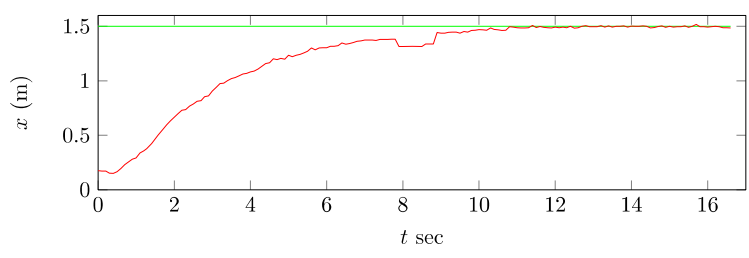

Figure 9: Position response of the cart

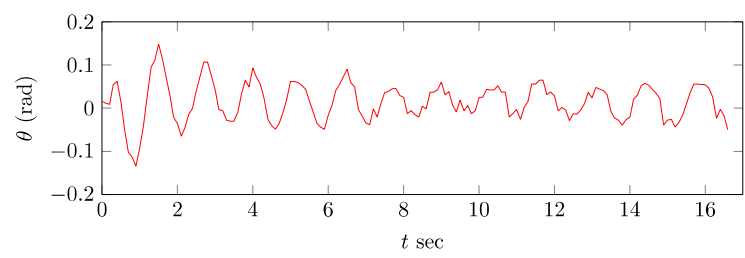

Figure 10: Swing angle response of the payload

Figure 11 and Figure 12 show respectively the control input generated by the controller and the sliding functions. Initially, the control force is around $2 \mathrm{~N}$. The chattering effect can be observed after the system has 
reached the equilibrium. Figure 11 shows the sliding surfaces of our proposed method. It can be seen that the sliding surfaces $S=0$ and consequently, $s_{1}=0$ and $s_{2}=0$ are controlled to reach the equilibrium in about $7 \mathrm{sec}$.

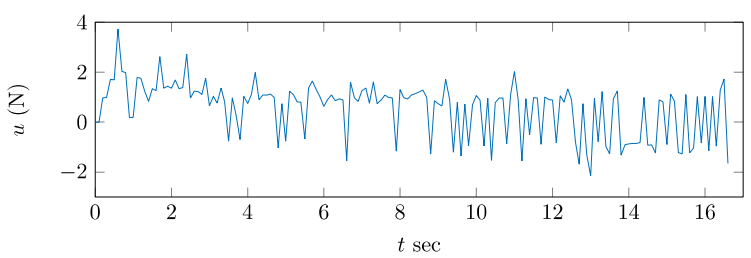

Figure11: Control input.

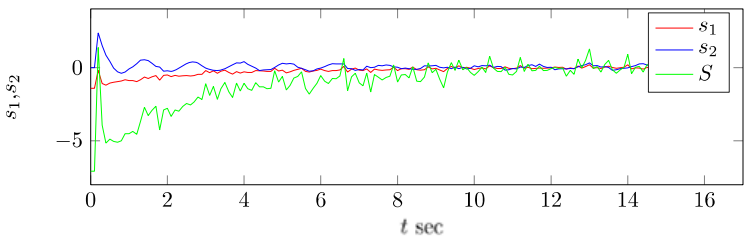

Figure 12: First and the second layer sliding surfaces

\section{Conclusion}

This paper has presented the design and implementation of a robust controller for gantry cranes. The control method is based on the Fast Terminal Sliding Mode. The proposed method provides enhanced convergence rate to the equilibrium in face of bounded uncertainties. Comparative study of the proposed method with the Terminal Sliding Mode verifies its advantage in simulation. Experiments were successfully conducted to validate the simulation results. The control performance obtained could be improved by using accurate sensors or estimation techniques. Furthermore, this method can be extended to other types of cranes such as those of boom type or offshore cranes.

\section{References}

[1] Raja Ismail, R. M. T., That, N. D. and Ha, Q. P. Modelling and robust trajectory following for offshore container crane systems. Automation in Construction. Vol. 59, pp. 179-187, 2015

[2] Ha, Q.P., Nguyen, M.T, Li, J. and Kwok, N.M. Smart structures with current-driven MR dampers: Modelling and second-order sliding mode control. IEEE/ASME Transactions on Mechatronics. 18-8, 1702-1712, 2013.

[3] Ngo Q. and Hong K. Adaptive sliding mode control of container cranes. IET control theory \& applications. Vol. 6, 662-668, 2012.

[4] Yu X. and Zhihong M. Multi-input uncertain linear systems with terminal sliding-mode control. Automatica. Vol. 34, 389-392, 1998.

[5] Liu J. and Wang X. Advanced Sliding Mode Control for Mechanical Systems, Springer, 2011.

[6] $\mathrm{Yu} \mathrm{X}$. and Zhihong M. Fast Terminal SlidingMode Control Design for Nonlinear Dynamical Systems. IEEE Trans. Circuits \& Systems I. Vol. 49, 261-264, 2002.

[7] Liu Y. and Yu H. A. Survey of underactuated mechanical systems. Control Theory \& Applications, IET, Vol. 7, 921-935, 2013.

[8] $\mathrm{Yu}, \mathrm{X}$ and Zhihong $\mathrm{M}$. On finite time mechanism: Terminal sliding modes. Proc. Int. Workshop on Variable Structure Systems. pp. 164-167, 1996.

[9] Zak, M. Terminal attractors for addressable memory in neural networks. Physics Letters A. Vol. 133, 18-22, 1988.

[10] Wang W., Yi J., Zhao D. and Liu D. Design of a stable sliding-mode controller for a class of second-order underactuated systems. IEE Proceedings: Control Theory and Applications. Vol. 151(6), 683-690, 2004. 